\title{
The third-order law for increments in magnetohydrodynamic turbulence with constant shear
}

\author{
M. Wan, ${ }^{1}$ S. Servidio, ${ }^{1}$ S. Oughton, ${ }^{2}$ and W. H. Matthaeus ${ }^{1}$ \\ ${ }^{1}$ Department of Physics and Astronomy and Bartol Research Institute, University of Delaware, Newark, \\ Delaware 19716, USA \\ ${ }^{2}$ Department of Mathematics, University of Waikato, Hamilton 3240, New Zealand
}

(Received 28 May 2009; accepted 8 September 2009; published online 29 September 2009)

\begin{abstract}
We extend the theory for third-order structure functions in homogeneous incompressible magnetohydrodynamic turbulence to the case in which a constant velocity shear is present. A generalization is found of the usual relation [Politano and Pouquet, Phys. Rev. E 57, 21 (1998)] between third-order structure functions and the dissipation rate in steady inertial range turbulence, in which the shear plays a crucial role. In particular, the presence of shear leads to a third-order law which is not simply proportional to the relative separation. Possible implications for laboratory and space plasmas are discussed. (C) 2009 American Institute of Physics. [doi:10.1063/1.3240333]
\end{abstract}

A well known result in hydrodynamic turbulence theory is the Kolmogorov-Yaglom ("4/5") law that relates the thirdorder structure function to the energy dissipation rate. ${ }^{1-3}$ Often regarded as a rigorous result of the fluid equations, this law requires assumptions of isotropy, homogeneity, and time stationarity of the statistics of velocity increments $\delta \boldsymbol{u}=\boldsymbol{u}(\boldsymbol{x}$ $+\boldsymbol{r})-\boldsymbol{u}(\boldsymbol{x})$ (velocity $\boldsymbol{u}$, spatial positions $\boldsymbol{x}+\boldsymbol{r}$ and $\boldsymbol{x}$ ). In addition - and crucially — it also requires adoption of the von Kármán hypothesis ${ }^{4}$ that the rate of energy dissipation $\epsilon$ approaches a constant nonzero value as the Reynolds number tends to infinity. Without the need for assuming isotropy, one finds

$$
\frac{\partial}{\partial r_{i}}\left\langle\delta u_{i}|\delta \boldsymbol{u}|^{2}\right\rangle=-4 \epsilon,
$$

where $\langle\cdots\rangle$ indicates an ensemble average and a sum on repeated indices is implied. If isotropy is further assumed, then

$$
\left\langle\delta u_{L}|\delta \boldsymbol{u}|^{2}\right\rangle=-\frac{4}{d} \epsilon|\boldsymbol{r}|,
$$

where $d$ is the number of spatial dimensions and $\delta u_{L}=\hat{\boldsymbol{r}} \cdot \delta \boldsymbol{u}$ is the increment component measured in the direction of the unit vector $\hat{\boldsymbol{r}}$ parallel to the relative separation $\boldsymbol{r}$. Extension of the third-order law to the case of incompressible magnetohydrodynamics (MHD) was reported in Ref. 5, which remained close to the approximations made in the hydrodynamic case. Without assuming isotropy, they found

$$
\frac{\partial}{\partial r_{i}}\left\langle\delta z_{i}^{\mp}\left|\delta z^{ \pm}\right|^{2}\right\rangle=-4 \epsilon^{ \pm},
$$

which, after adoption of isotropy, reduces to

$$
\left\langle\delta z_{L}^{\mp}\left|\delta z^{ \pm}\right|^{2}\right\rangle=-\frac{4}{d} \epsilon^{ \pm} r,
$$

where $\delta z^{ \pm}=z^{ \pm}(\boldsymbol{x}+\boldsymbol{r})-z^{ \pm}(\boldsymbol{x})$ are the increments of the Elsässer variables and $\delta z_{L}^{ \pm}=\hat{\boldsymbol{r}} \cdot \delta z^{ \pm}$. The constants $\epsilon^{ \pm}$are the mean energy dissipation rates of the corresponding variables $\boldsymbol{z}^{ \pm}=\boldsymbol{u} \pm \boldsymbol{b}$, where $\boldsymbol{b}$ is the magnetic field fluctuation in Alfvén speed units.

Here we extend the third-order law in MHD turbulence to cases in which the isotropy assumption is relaxed. This is accomplished by introducing homogeneous shear in the velocity field, a simplified and well-studied approach in hydrodynamics. $^{6-9}$ In particular, it supports departures from strict isotropy and introduction of shear without consideration of rigid boundaries. MHD third-order laws have been applied to systems that may also admit departures from strict uniformity due to coherent large-scale gradients, e.g., plasma confinement devices ${ }^{10,11}$ and the solar wind. ${ }^{12-15}$ For systems like these, the homogeneous shear approximation may be a reasonable step toward including such large-scale effects in the relevant MHD turbulence scaling laws. To this end, our derivation of the MHD third-order law will include the effect of homogeneous shear, leading to a necessarily anisotropic form for the law.

More specifically, we find that a uniform shear introduces new terms in the third-order law, so that one can no longer conclude that a particular third-order structure function, or even a particular integral of a third-order structure function, is proportional to the dissipation rate times the relative separation length $r$. This is in marked contrast to the situation for the fully isotropic hydrodynamic and MHD cases, given here as Eqs. (2) and (4). It is, however, entirely consistent with the work in Refs. 16 and 17, in which modifications to the form of the third-order law for hydrodynamics with shear were derived.

The principal theoretical result given below is that a uniform shear indeed is responsible for changing the form of the third-order law, whereas a mean magnetic field does not produce such structural changes. Implications for solar wind, laboratory, and astrophysical measurements of turbulence are suggested, and, in particular, the primacy of the third-order law in unambiguously defining an inertial range is challenged.

The third-order law is often derived from the steadystate version of an equation related to energy decay. To ob- 
tain the version of the law appropriate for MHD with uniform velocity shear, we follow the same procedure used previously for MHD, ${ }^{5,13}$ combined with the method in Ref. 17 for extending Eq. (2) to include shear. A uniform magnetic field is also retained, although only the simplest of its consequences will enter the discussion.

First, let us employ a Reynolds decomposition of the velocity field $\boldsymbol{v}=\boldsymbol{U}+\boldsymbol{u}$ into a mean velocity $\boldsymbol{U}(\boldsymbol{x})$ and a fluctuating component $\boldsymbol{u}(\boldsymbol{x}, t)$, where $\langle\boldsymbol{v}\rangle=\boldsymbol{U}$ and $\langle\boldsymbol{u}\rangle=\mathbf{0}$. Similarly we write the total magnetic field, conveniently expressed in Alfvén speed units, as $\boldsymbol{B}=\boldsymbol{b}+\boldsymbol{B}_{0}$. We assume $\boldsymbol{B}_{0}$ is constant and uniform but that $\boldsymbol{U}(\boldsymbol{x})$ varies in space. However, this variation will be taken as nonrandom and slowly varying, so that the turbulence properties can be treated as locally homogeneous.

Now we write the incompressible MHD equations at two positions, $\boldsymbol{x}$ and $\boldsymbol{x}^{\prime}=\boldsymbol{x}+\boldsymbol{r}$,

$$
\begin{aligned}
\partial_{t} z_{i}^{ \pm}= & -\left(z_{k}^{\mp}+U_{k} \mp B_{0 k}\right) \partial_{k}\left(U_{i}+z_{i}^{ \pm}\right)-\partial_{i} P+\nu \partial_{k} \partial_{k} z_{i}^{ \pm}, \\
\partial_{t} z_{i}^{ \pm \prime}= & -\left(z_{k}^{\mp \prime}+U_{k}^{\prime} \mp B_{0 k}\right) \partial_{k}^{\prime}\left(U_{i}^{\prime}+z_{i}^{ \pm \prime}\right)-\partial_{i}^{\prime} P^{\prime} \\
& +\nu \partial_{k}^{\prime} \partial_{k}^{\prime} z_{i}^{ \pm \prime} .
\end{aligned}
$$

Here the prime denotes quantities at position $\boldsymbol{x}^{\prime}, P$ is the pressure, and $\nu$ is the kinematic viscosity, taken equal to the resistivity hereafter. Subtracting Eq. (5) from Eq. (6) yields the following equation for the Elsässer increments $\delta z^{ \pm}$ $=z^{ \pm}\left(x^{\prime}\right)-z^{ \pm}(x)$ :

$$
\begin{aligned}
\partial_{t} \delta z_{i}^{ \pm}= & -\left(\delta U_{k}+\delta z_{k}^{\mp}\right) \partial_{k}^{\prime} \delta z_{i}^{ \pm} \\
& -\left(z_{k}^{\mp}+U_{k} \mp B_{0 k}\right)\left(\partial_{k}^{\prime}+\partial_{k}\right) \delta z_{i}^{ \pm} \\
& -\left(\delta z_{k}^{\mp}+\delta U_{k}\right) \partial_{k} U_{i}-\left(z_{k}^{\mp \prime}+U_{k}^{\prime} \mp B_{0 k}\right) \delta\left(\partial_{k} U_{i}\right) \\
& -\left(\partial_{i}^{\prime}+\partial_{i}\right) \delta P+\nu\left(\partial_{k}^{\prime} \partial_{k}^{\prime}+\partial_{k} \partial_{k}\right) \delta z_{i}^{ \pm},
\end{aligned}
$$

where we use the property that the primed and unprimed coordinates are independent, so that $\partial_{k} z_{i}^{ \pm \prime}=0$ and $\partial_{k}^{\prime} z_{i}^{ \pm}=0$.

As noted above, we seek an equation related to energy decay. Multiplying the previous equation by $2 \delta z_{i}^{ \pm}$and averaging yields

$$
\begin{aligned}
\left.\left.\partial_{t}|| \delta z_{i}^{ \pm}\right|^{2}\right\rangle= & -\frac{\partial}{\partial r_{k}}\left\langle\left(\delta U_{k}+\delta z_{k}^{\mp}\right)\left|\delta z_{i}^{ \pm}\right|^{2}\right\rangle \\
& +\left\langle\left|\delta z_{i}^{ \pm}\right|^{2}\left(\partial_{k} U_{k}+\partial_{k}^{\prime} U_{k}^{\prime}\right)\right\rangle \\
& -2\left\langle\partial_{k} U_{i} \delta z_{i}^{ \pm}\left(\delta z_{k}^{\mp}+\delta U_{k}\right)\right\rangle \\
& -2\left\langle\left(z_{k}^{\mp \prime}+U_{k}^{\prime} \mp B_{0 k}\right) \delta\left(\partial_{k} U_{i}\right) \delta z_{i}^{ \pm}\right\rangle \\
& +2 \nu \frac{\partial^{2}}{\partial r_{k}^{2}}\left\langle\left|\delta z_{i}^{ \pm}\right|^{2}\right\rangle-4 \nu\left\langle\left|\partial_{k} z_{i}^{ \pm}\right|^{2}\right\rangle .
\end{aligned}
$$

In arriving at this expression we make use of $\partial_{k}\langle\cdot\rangle$ $=-\left(\partial / \partial r_{k}\right)\langle\cdot\rangle$ and $\partial_{k}^{\prime}\langle\cdot\rangle=\left(\partial / \partial r_{k}\right)\langle\cdot\rangle$. These latter relations follow from spatial homogeneity (i.e., translation invariance of the statistical properties), which can be considered for some systems to be an exact property (see below) or an approximation, e.g., in the case of a weakly inhomogeneous system. The main results here will be for strict homogeneity.

The last term of Eq. (8) can be identified with the dissipation rates

$$
\epsilon^{ \pm}=\nu\left\langle\left|\partial_{k} z_{i}^{ \pm}\right|^{2}\right\rangle,
$$

which for steady state are also the mean energy transfer rates. Following the usual arguments, ${ }^{4}$ in the limit of vanishing viscosity $\nu \rightarrow 0$, it is assumed-not proven - that the $\epsilon^{ \pm}$ remain nonzero and in effect are externally prescribed by the rate of supply of turbulence energy (and cross helicity). Although this nontrivial assertion is physically plausible, ${ }^{18}$ it nonetheless prevents the subsequent developments, including the classical 4/5 law, from being considered an exact consequence of the fluid equations themselves. Furthermore, the penultimate term in Eq. (8), also involving the viscosity, is assumed to vanish at high Reynolds number when we are examining the inertial range of separations. For the abovestated set of approximations, the increments $r$ are restricted to lie in the inertial range, that is, separations smaller than the correlation length (energy-containing scale) and bigger than the dissipation scale (scale at which fluctuations are critically damped). For variations of the set of assumptions that lead to a third-order law, see, e.g., Ref. 19.

The above relations need not be strictly homogeneous, as variations in $\boldsymbol{U}$ over the slowly varying large scales may be present. To rectify this and arrive at a general law that is translation invariant, we now specialize to the case of a homogeneous shear flow, alluded to earlier. With this choice the tensor $\partial U_{i} / \partial x_{j}$ is a constant matrix independent of position. The turbulence is then homogeneous and all terms in Eq. (8) — both coefficients and averaged terms - are only a function of the separation vector $\boldsymbol{r}$.

Under the hypothesis of steady-state turbulence, the lefthand side of Eq. (8) vanishes. Integrating in $\boldsymbol{r}$, over a volume $\mathcal{V}$ that is enclosed by a surface $\mathcal{S}$, the equation becomes

$$
\begin{aligned}
\oint_{\mathcal{S}} & {\left[\hat{n}_{k}\left\langle\left(\delta z_{k}^{\mp}+\delta U_{k}\right)\left|\delta z_{i}^{ \pm}\right|^{2}\right\rangle\right] d S+2 \frac{\partial U_{i}}{\partial x_{k}} \int_{\mathcal{V}}\left\langle\delta z_{i}^{ \pm} \delta z_{k}^{\mp}\right\rangle d V } \\
= & -4 V \epsilon^{ \pm},
\end{aligned}
$$

where $V$ is the volume of the region $\mathcal{V}, d S$ is the differential area on $\mathcal{S}$, and $\hat{n}_{k}$ is a unit vector normal to $\mathcal{S}$.

If the region of integration is a three dimensional sphere of radius $r$, volume $V_{r}$, and surface $S_{r}$, the integration yields

$$
S_{3}^{ \pm}+S_{U}^{ \pm}+S_{\alpha}^{ \pm}=-\frac{4}{3} \epsilon^{ \pm} r,
$$

where

$$
\begin{aligned}
& S_{3}^{ \pm} \equiv \frac{1}{4 \pi r^{2}} \oint\left\langle\delta z_{L}^{\mp}\left|\delta z_{i}^{ \pm}\right|^{2}\right\rangle d S, \\
& S_{U}^{ \pm} \equiv \frac{1}{4 \pi r^{2}} \oint\left\langle\delta U_{L}\left|\delta z_{i}^{ \pm}\right|^{2}\right\rangle d S, \\
& S_{\alpha}^{ \pm} \equiv \frac{1}{2 \pi r^{2}} \frac{\partial U_{i}}{\partial x_{k}} \int\left\langle\delta z_{i}^{ \pm} \delta z_{k}^{\mp}\right\rangle d V,
\end{aligned}
$$

where, again, $\delta z_{L}^{ \pm}=\hat{\boldsymbol{r}} \cdot \delta z^{ \pm}$and $\delta U_{L}=\hat{\boldsymbol{r}} \cdot \delta \boldsymbol{U}$. And now in spherical $\left(r^{\prime}, \theta, \phi\right)$ coordinates $d S=r^{2} d(\cos \theta) d \phi \equiv r^{2} d \Omega$ and $d V=r^{\prime 2} d \Omega d r^{\prime}$. Equation (11) may be interpreted as the integral form of the third-order law for incompressible homogeneous MHD turbulence with an external velocity field that is 
constant in time but which can vary linearly in space. By setting $\boldsymbol{U}=\mathbf{0}$ and assuming isotropic turbulence, Eq. (11) will recover the standard third-order law for isotropic MHD turbulence, ${ }^{5}$ given here as Eq. (4).

In standard derivations for isotropic turbulence, ${ }^{5,13,14}$ shear is necessarily lacking, and it is assumed that the structure functions are rotationally symmetric. In that case the above relation is simplified by carrying out the integrals explicitly. (For a more general case, see below.) Here we allow for anisotropy induced either by a uniform large-scale magnetic field or by an imposed homogeneous shear. Note that a uniform magnetic field $\boldsymbol{B}_{0}$ has increments $\delta \boldsymbol{B}_{0}=\mathbf{0}$, and thus it does not appear explicitly in the third-order relation, even though it is well documented that such a field induces spectral anisotropy in MHD turbulence. ${ }^{20}$

We now further specialize to the large-scale homogeneous shear flow $\boldsymbol{U}=U_{x}(y) \hat{\boldsymbol{x}}=\alpha y \hat{\boldsymbol{x}}$ in a Cartesian $(x, y, z)$ system, with $\alpha=$ const. The integral form of the third-order relation, in the form $S_{3}^{ \pm}=-S_{U}^{ \pm}-S_{\alpha}^{ \pm}-\frac{4}{3} \epsilon^{ \pm} r$, becomes

$$
\begin{aligned}
\left\langle\left\langle\left.\delta z_{L}^{\mp}\left|\delta z^{ \pm}\right|\right|^{2}\right\rangle\right\rangle_{\Omega}= & -\alpha r\left\langle\left\langle\left.(\hat{\boldsymbol{r}} \cdot \hat{\boldsymbol{x}})(\hat{\boldsymbol{r}} \cdot \hat{\boldsymbol{y}})\left|\delta z^{ \pm}\right|\right|^{2}\right\rangle\right\rangle_{\Omega} \\
& -\frac{2}{3} \alpha r\left\langle\left\langle\delta z_{x}^{ \pm} \delta z_{y}^{\mp}\right\rangle\right\rangle_{V}-\frac{4}{3} r \epsilon^{ \pm},
\end{aligned}
$$

denoting an angular average over a shell of radius $r$ as $\langle\cdots\rangle_{\Omega}$ and a volume average over a sphere of radius $r$ as $\langle\cdots\rangle_{V}$. This form, based on a spherical region of radius $r$, indicates that all three terms on the right-hand side of the equation have an explicit proportionality to $r$; moreover, the first and second of these also admit an implicit dependence on $r$. The quantity on the left side of Eq. (13) is the MHD analog of the usual third-order structure function that appears in the Yaglom and Kolmogorov laws, ${ }^{1,2}$ and we see that in the presence of homogeneous shear it is not simply proportional to the dissipation $\epsilon^{ \pm}$. Instead, under the stated assumptions, only the sum of the first three terms in Eq. (13) is guaranteed to be proportional to the dissipation rate.

At this point we remark on an alternative form that the third-order law can assume that may be revealing in anisotropic cases. Recall that Eq. (10) is valid for an arbitrary volume $\mathcal{V}$ and its associated bounding surface $\mathcal{S}$. The advantage of employing a spherical volume $\mathcal{V}$ is that when the flux is isotropic, the integrand in the surface integral will be independent of the direction of $\boldsymbol{r}$, making the integration trivial. Unfortunately, this property is lost when the turbulence is anisotropic. ${ }^{20,21}$ However, provided that the (energylike) vector flux $\boldsymbol{F}^{+}=\left\langle\left(\delta \boldsymbol{z}^{-}+\delta \boldsymbol{U}\right)\left|\delta \boldsymbol{z}^{+}\right|^{2}\right\rangle$ is smoothly varying in $\boldsymbol{r}$, it is in principle possible to find a set of nested surfaces $\mathcal{S}(\mathcal{V})$ (labeled by their enclosing volume $V$ and with unit normal vectors $\hat{\boldsymbol{n}}_{\mathcal{S}}$ ), such that the normal component of the vector flux $\boldsymbol{F}^{+}$is uniform across $\mathcal{S}(\mathcal{V})$. Then $\oint_{\mathcal{S}} d S \hat{\boldsymbol{n}}_{\mathcal{S}} \cdot \boldsymbol{F}^{+}$ $=F_{n}^{+}(V) S$, where the constant normal flux $F_{n}$ is labeled by the volume $V$ bounded by the surface, and $S$ is the value of the surface area. The partner quantity $\boldsymbol{F}^{-}$is defined analogously. Provided these nested surfaces can be found, the homogeneous shear case, Eq. (10), can then be reduced to

$$
\begin{aligned}
F_{n}^{ \pm}\left(V^{ \pm}\right) & =\left\langle\left.\left[\hat{\boldsymbol{n}}_{\mathcal{S}^{ \pm}} \cdot\left(\delta z^{\mp}+\delta \boldsymbol{U}\right)\left|\delta z^{ \pm}\right|^{2}\right]\right|_{\mathcal{S}^{ \pm}}\right\rangle \\
& =-\frac{2 \alpha V^{ \pm}}{S^{ \pm}}\left\langle\left\langle\delta z_{x}^{ \pm} \delta z_{y}^{\mp}\right\rangle\right\rangle_{\mathcal{V}}-\frac{4 V^{ \pm}}{S^{ \pm}} \epsilon^{ \pm},
\end{aligned}
$$

where $V^{ \pm}$and $S^{ \pm}$are the volumes and associated surface areas that admit constant normal fluxes $F_{n}^{ \pm}\left(V^{ \pm}\right)$. Note that in general the constant flux surfaces $S^{+}$and $S^{-}$are expected to be different, e.g., due to cross helicity effects.

When homogeneous shear is absent the result in Eq. (14) reduces to the formal anisotropic third-order law

$$
F_{n}^{ \pm}\left(V^{ \pm}\right)=-\frac{4 V^{ \pm}}{S^{ \pm}} \epsilon^{ \pm}
$$

The latter can have application in the cases in which anisotropy is present due to a mean magnetic field $\boldsymbol{B}_{0} \neq \mathbf{0}$.

In summary, we examined the mixed third-order Elsässer structure functions for MHD turbulence, incorporating a constant sheared velocity (homogeneous shear) field in addition to homogeneous fluctuations, under a set of assumptions that parallel those used in standard turbulence theory to derive the Kolmogorov 4/5 law. In analogy to the findings in Refs. 16 and 17 for hydrodynamics, we find that a law can be obtained for stationary homogeneous turbulence that relates third-order structure functions and dissipation but which also involves additional terms. For MHD with a constant imposed shear, there are shear-related terms that appear in this modified third-order law, as in the hydrodynamic case. On the other hand, a uniform magnetic field does not appear explicitly in this relation, as it does not contribute directly to the increments.

On the basis of a very simple estimate we expect the new terms in the third-order equation to be of significance when the large-scale velocity increments are of the same order or larger than the fluctuation increments at the same separation $r$, that is, when $\delta U \sim \delta z$. In some applications this condition may be realized, and consequently the classical third-order law is modified by these new terms. We suspect that for solar wind turbulence, as well as for laboratory devices, the present generalized form of the third-order law will be relevant. Estimating both $\left\langle\left|\delta z^{ \pm}\right|^{2}\right\rangle$ and $\left\langle z_{x}^{+} z_{y}^{-}\right\rangle$as $\sim r^{2 / 3}$, one expects that over a limited inertial range, $S_{U}^{ \pm}$and $S_{\alpha}^{ \pm}$will scale as $\sim r^{5 / 3}$. Therefore we expect that these additional terms have the potential to dominate the summation in Eq. (11), especially at large scales, even though the full sum should always be $\sim r$. However, in all cases in which the terms $S_{U}^{ \pm}$and $S_{\alpha}^{ \pm}$cannot be neglected, the modified MHD third-order law no longer admits an interpretation purely in terms of energy transfer and dissipation, and therefore differs from the isotropic case without shear.

Several other extensions of the third-order law result in formulas similar to those above. For example, including a large-scale magnetic shear is straightforward and can be included in place of the velocity shear in Eqs. (11)-(13) by the substitutions $\delta U_{L} \rightarrow \mp \delta B_{L}$ and $\partial U_{i} / \partial x_{k} \rightarrow \pm \partial B_{i} / \partial x_{k}$ in Eq. (12). The combined case of velocity and magnetic shear is 
accomplished by setting $\delta U_{L} \rightarrow \delta Z_{L}^{\mp}$ and $\partial U_{i} / \partial x_{k} \rightarrow \partial Z_{i}^{ \pm} / \partial x_{k}$ in Eq. (12), where the large-scale Elsässer fields are $Z_{k}^{ \pm}$ $=U_{k} \pm B_{k}$ in Alfvén speed units. Still another extension is to the case of uniform rotation, accomplished by setting $\partial U_{i} / \partial x_{k} \rightarrow \frac{1}{2} \epsilon_{i k m} \Omega_{m}$, an antisymmetric tensor with $\Omega$ the uniform rate of rotation. (Here $\epsilon_{i k m}$ is the Levi-Civita symbol.)

As a final remark, we note that the modifications of the third-order law for energy decay that we describe here can be anticipated in the structure of scale-separated transport equations derived for MHD in a weakly inhomogeneous medium. ${ }^{22,23}$ These two-scale transport equations provide a formalism for evolution of second-order correlation functions and include nonlinear decay, analogous to our thirdorder structure functions, along with advection and shear terms. On this basis, one could have already concluded that the third-order law requires modification in the presence of large-scale shear. The present study concentrated only on the special case of homogeneous shear, and generalizations of the third-order law have been found.

We expect that future studies based on numerical simulations may provide explicit verification and examples of the relationships we propose here. Taking into account effects such as shear, observational studies may prove useful in a variety of systems with large-scale shear flows, such as astrophysical and laboratory plasmas.

This research was supported in part by the NSF Solar Terrestrial Program ATM0539995 and SHINE ATM0752135 and by NASA under Heliophysics Theory Program grant NASA NNX08AI47G.
${ }^{1}$ A. N. Kolmogorov, C. R. Acad. Sci. URSS 32, 16 (1941); reprinted in Proc. R. Soc. London, Ser. A 434, 15 (1991).

${ }^{2}$ A. S. Monin and A. M. Yaglom, Statistical Fluid Mechanics (MIT Press, Cambridge, 1971), Vol. 1; A. S. Monin and A. M. Yaglom, Statistical Fluid Mechanics (MIT Press, Cambridge, 1975), Vol. 2.

${ }^{3}$ U. Frisch, Turbulence (CUP, Cambridge, 1995).

${ }^{4}$ T. de Kármán and L. Howarth, Proc. Roy. Soc. London, Ser. A 164, 192 (1938).

${ }^{5}$ H. Politano and A. Pouquet, Geophys. Res. Lett. 25, 273 (1998).

${ }^{6}$ R. S. Rogallo, NASA Technical Memorandum No. TM-81315, 1981.

${ }^{7}$ M. J. Lee, J. Kim, and P. Moin, J. Fluid Mech. 216, 561 (1990).

${ }^{8}$ S. Kida and M. Tanaka, J. Fluid Mech. 274, 43 (1994).

${ }^{9}$ A. Pumir, Phys. Fluids 8, 3112 (1996).

${ }^{10}$ G. Serianni, M. Agostini, V. Antoni, R. Cavazzana, E. Martines, F. Sattin, P. Scarin, E. Spada, M. Spolaore, N. Vianello, and M. Zuin, Plasma Phys. Controlled Fusion 49, B267 (2007)

${ }^{11}$ F. Lepreti, V. Carbone, M. Spolaore, V. Antoni, R. Cavazzana, E. Martines, G. Serianni, P. Veltri, N. Vianello, and M. Zuin, Europhys. Lett. 86, 25001 (2009).

${ }^{12}$ C.-Y. Tu and E. Marsch, Space Sci. Rev. 73, 1 (1995).

${ }^{13}$ L. Sorriso-Valvo, R. Marino, V. Carbone, A. Noullez, F. Lepreti, P. Veltri, R. Bruno, B. Bavassano, and E. Pietropaolo, Phys. Rev. Lett. 99, 115001 (2007).

${ }^{14}$ B. T. MacBride, C. W. Smith, and M. A. Forman, Astrophys. J. 679, 1644 (2008).

${ }^{15}$ R. Marino, L. Sorriso-Valvo, V. Carbone, A. Noullez, R. Bruno, and B. Bavassano, Astrophys. J. Lett. 677, L71 (2008).

${ }^{16}$ E. Lindborg, J. Fluid Mech. 326, 343 (1996).

${ }^{17}$ C. M. Casciola, P. Gualtieri, R. Benzi, and R. Piva, J. Fluid Mech. 1476, 105 (2003).

${ }^{18}$ G. L. Eyink and K. R. Sreenivasan, Rev. Mod. Phys. 78, 87 (2006).

${ }^{19}$ R. J. Hill, J. Fluid Mech. 353, 67 (1997).

${ }^{20}$ J. V. Shebalin, W. H. Matthaeus, and D. Montgomery, J. Plasma Phys. 29, 525 (1983).

${ }^{21}$ B. Teaca, M. K. Verma, B. Knaepen, and D. Carati, Phys. Rev. E 79, 046312 (2009).

${ }^{22}$ C.-Y. Tu and E. Marsch, J. Plasma Phys. 44, 103 (1990).

${ }^{23}$ Y. Zhou and W. H. Matthaeus, J. Geophys. Res. 95, 10291, doi:10.1029/ JA095iA07p10291 (1990). 